(RESEARCH ARTICLE)

\title{
Diversity and abundance of macrophytes of streams under different anthropogenic influences in the Buea municipality, southwestern Cameroon
}

\author{
Anyinkeng Neculina $1,{ }^{*}$, Mih Mathias Afui ${ }^{1}$ and Tening Aaron Suh ${ }^{2}$ \\ ${ }^{1}$ Department of Botany and Plant Physiology, Faculty of Science, University of Buea, P. O Box 63, Buea, Cameroon. \\ 2 Department of Agronomic and Applied Molecular Sciences, Faculty of Agriculture and Veterinary Medicine, University of \\ Buea, P. O Box 63, Buea, Cameroon.
}

Publication history: Received on 30 December 2019; revised on 08 January 2020; accepted on 09 January 2020

Article DOI: https://doi.org/10.30574/wjarr.2020.5.1.0112

\begin{abstract}
Macrophytes can occur as submerged, floating or emergent. They provide not only important structural supports in streams and river habitats, but also absorb and sequester pollutants, provide primary food production, nutrients and habitats to a wide range of macro and micro-organisms living in and around lotic sites. Their existence is however threatened by both anthropogenic and natural stresses. This study aimed to document macrophyte diversity and abundance in streams under different anthropogenic influences in Buea, southwestern Cameroon. Floristic surveys were carried out using $500 \mathrm{~m}$ long transects laid along the different streams. Plants encountered were identified and confirmed at the Limbe Botanic Garden Herbarium. One hundred and ten species belonging to 38 families and 83 genera were recorded. There were no floating species and only one stream had submerged species (Vallisneria spiralis L and Crinum calamistratum Bogner \& Heine). The highest number of families (26), genera (58) and species (70) were obtained in Ndongo while the least of these taxa were obtained in Bulu (16, 35 and 39, respectively). Overall, Asteraceae and Poaceae were the most abundant, suggesting anemophilic dispersals. However, individual species abundances varied with streams. The highest diversity index (0.945) was in Wongangjio while the least (0.760) was in Nange, suggesting that waste discharge from car wash might influence species diversity negatively. The macrophyte composition was made up of obligate and non-obligate species as a consequence of anthropogenic influences. These results constitute baseline data for the area that can be exploited for further research and monitoring.
\end{abstract}

Keywords: Macrophyte; Streams; Anthropogenic influence; Buea Municipality

\section{Introduction}

Aquatic ecosystems usually contain macrophytes which may be submerged, floating or emergent. The plants are very important in the structure and function of the aquatic system in that they absorb and sequester pollutants, reduce erosion by damping wave action and stabilize shorelines and river bottoms [1,2,3]. Additionally, macrophytes provide primary food production, nutrients and habitat for a wide range of macro and micro-organisms living in and around lotic sites [4]. The effectiveness of their functioning depends, amongst other factors, on their diversity and abundance [5].

Studies on macrophyte abundance and diversity in Cameroon are rare $[1,6,7]$. Other studies involving macrophytes in other areas of the country, focus on their use in wastewater treatment $[8,9,10]$. Little or no such data exist for the Southwest Region and Buea in particular.

Buea lies between $3^{0} 57^{\prime} \mathrm{N}-4^{0} 27^{\prime} \mathrm{N}$ and $8^{0} 58^{\prime}-9^{0} 5^{\prime} \mathrm{E}$ on the eastern flank of mount Cameroon. The mean annual precipitation and temperature stand at $3000 \mathrm{~mm}$ and $28{ }^{\circ} \mathrm{C}$, respectively. The mean relative humidity is $86 \%$ and

\footnotetext{
${ }^{*}$ Corresponding author

E-mail address: anyinkengnicoline@yahoo.com
}

Copyright (C) 2020 Author(s) retain the copyright of this article. This article is published under the terms of the Creative Commons Attribution Liscense 4.0. 
sunshine ranges from 900 to 1200 hrs per annum [11]. The climate is equatorial, with two seasons: a dry season from November to February and a rainy season from March to October. The municipality has a rich hydrological network. The absence of conscious efforts to protect water catchments, haphazard waste disposal especially in water ways, deforestation motivated by agriculture, timber for local consumption, fuel wood and bush fires (natural and hunting fires) have all contributed to degrade the aquatic ecosystem within the municipality.

This work had as objective to assess the diversity and abundance of macrophytes from five streams with different anthropogenic influences in the Buea municipality. This baseline data will complement data from other areas which can be exploited for further research and by aquatic ecosystem protection services of the municipality.

\section{Material and methods}

\subsection{Sampled streams and their characteristics}

Five streams subjected to different anthropogenic influences were selected. Farming as an activity cuts across the different streams.

Nange water: This is a stream located far from habitation but with characteristic that cars are driven into it and washed. In this stream, there are two car wash points.

2) Stream at Mile 17 Hill (M17): The catchment area of this stream was used as dumping ground for municipal waste before the arrival of the waste collection company in 2010. In this stream, cars are washed by the banks, with effluents discharged into it. It also receives storm drainage from Molyko, a residential neighbourhood with dense population.

3) Ndongo: It is exposed to waste from various anthropogenic (waste disposal, bathing and laundry) activities as it flows from lower Bokoko to Mile 16, passing through Molyko, with dense population. Despite the presence of the waste collection company, municipal waste are deposited at various points along the course of the stream. Bathing and laundry, in addition to farming, are other activities along the stream course.

4) Bulu water: It originates from Lower Bunduma and flows down to Bulu Native, with storm drainage as well as municipal wastes from Upper Bunduma and Great Soppo getting into it at various points along the course.

5) Wongangjio: It is a stream located far from habitation with no waste dump and no washing of cars`.

\subsection{Data collection}

Floristic inventory was carried out along 500 m long transects laid along the different streams (Figure 1). The transect for Nange originated $10 \mathrm{~m}$ before the first car wash point and extended downstream beyond the second car wash point. In the stream at Mile $17 \mathrm{Hill}$, the transect originated from the catchment and extended downstream beyond the car wash area. The transects for Ndongo and Wonganjio originated $10 \mathrm{~m}$ from the catchment. For Bulu, the transect was laid 10 $\mathrm{m}$ after the observed point of water emergence along the course.

Ten plots were laid per transect. The plots began $10 \mathrm{~m}$ from transect origin and were interspaced by a distance of $42 \mathrm{~m}$. Within each plot, sampling units of $1 \mathrm{mx} 1 \mathrm{~m}$ were mapped out by dividing it into strips of $(1 \mathrm{x} 1) \mathrm{m}$ and numbering. Ballots were then drawn to select sampling units such that $1 / 3$ of them were on each of the right and left bank and within the stream. A total of twelve sampling units were randomly selected within each plot. A frame measuring (1 x 1 $\mathrm{m}$, in-to-in) was placed at the selected number of the strip and the vegetation within it censured.

Plants were identified in the field. Unidentified species were photographed, voucher specimens collected and identified using illustrations and photographs in manuals and later confirmed at the Limbe Botanic Garden Herbarium (SCA). 


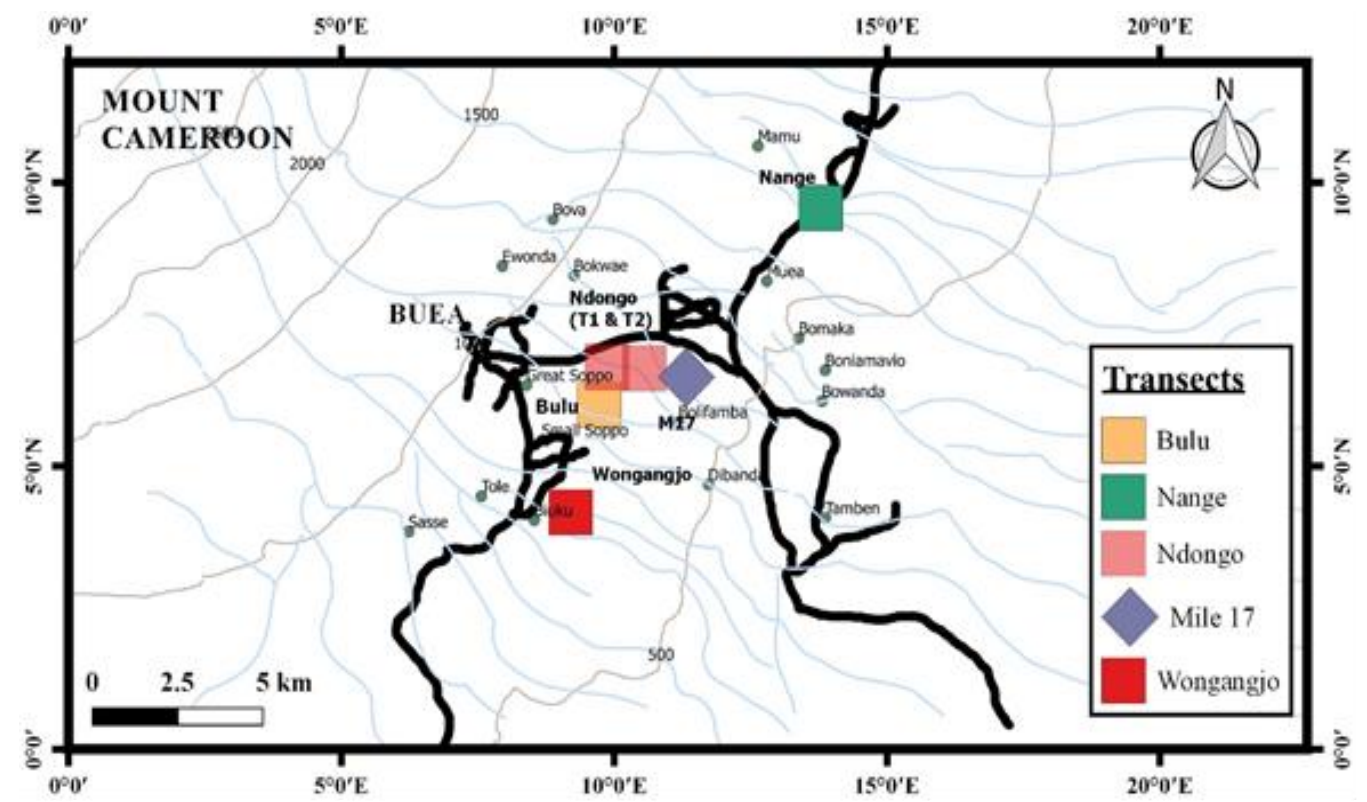

Figure 1 Location of transects for macrophyte assessment in different streams in Buea municipality

The frequency of occurrence and abundance of each species were recorded. Creeping species were evaluated by percent cover and later converted to count using Braun Blanquet scale index [12].

\subsection{Data analysis}

Species composition for the streams were determined through Simpson's diversity indices. Simpson's diversity index $=$ $1-\mathrm{D}$

Where;

$D=\sum\left(\frac{n_{i}}{N}\right)^{2}$

$n_{i}=$ number of individuals of species $\mathrm{i}$

$N=$ Total number of individuals of all species

Species richness was determined using the Menhinicks index (D)

$D=\frac{S}{\sqrt{N}}$

Where

$\mathrm{S}=$ number of different species in the sample

$\mathrm{N}=$ total number of individual organisms in the sample

Species similarity between steams was determined using Sörensen similarity coefficient (Ss).

$S_{S}=\frac{2 a}{2 a+b+c}$

Where $\mathrm{S}_{\mathrm{s}}=$ Sorenson similarity coefficient 


$$
\begin{aligned}
& a=\text { number of species common to all sites/category } \\
& b=\text { number of species unique to first site/category } \\
& c=\text { number of species unique to second site/category }
\end{aligned}
$$

\section{Results and discussion}

The study revealed a total of 108 emergent and two submerged (Vallisneria spiralis L. and Crinum calamistratum Bogner \& Heine, unique to Nange) species from the five streams. These species belong to 83 genera in 38 families (Table 1; Plates $1, \mathrm{~A}-\mathrm{F}$ ). No floating species was found in any of the streams. The absence of floating species is due to the sloppy nature of the streams since such species are characteristic of stagnant and slow-flowing waters. However, the peculiarity of Nange with regard to submerged species is probably due to the fact that it is the only stream in the list with gentle slope and wide area ( $40 \mathrm{~m}$ stream breadth). These characteristics give it the ability to accumulate sediments and nutrients thus allowing shallow pools of water which favour the establishment of submerged species.

The occurrence and abundance of the different taxa varied among streams (Fig 2). In Nange, there were a total of forty eight species. Asteraceae and Poaceae had the highest number of genera with six each while those with one genus included; Amaryllidaceae, Arecaceae, Brassicaceae, Commelinaceae, Crassulaceae, Lamiaceae and Tiliaceae. Vallisneria spiralis had the highest (40.98) relative abundance while species with the least (0.01) were; Asystasia gangetica, Raphia farinifera, Conyza boriensis, Vernonia amydalina and Plectranthus monostachyus.

In the stream at M17 hill with forty eight species, the Asteraceae had the highest number of genera (6) while Commelinaceae, Costaceae, Cyperaceae, Heliconiaceae, Malvaceae, Mimosaceae and Piperaceae were among the list of families represented by single genus. The species with the highest (31.06) relative abundance was Echinochloa pyramidalis while the least (0.04) included Anubias barteri, V. amydalina and Abelmoschus esculentus

Ndongo had seventy species. Poaceae had the highest genera (11) while families with single genus were many and included Asparagaceae, Cannaceae, Costaceae and Sellaginellaceae. Pennesetum purpureum had the highest (17.24) relative abundance while the least (0.03) were species such as Axonopus compressus, Colocasia esculenta and Diplocyclos palmatus

In Bulu, there were a total of thirty nine species and the families with the highest genera (6) were Poaceae and Asteraceae while those with the least (1) included Costaceae, Dryopteridaceae, Fabaceae, Oxalidaceae, Piperaceae and Solanaceae. P. purpureum had the highest (10.66) relative abundance while the least (0.08) was Oxalis simplex

In Wongangjio with a total of forty six species, Asteraceae had the highest (7) genera while the least were families such as Araceae, Brassicaceae, Blechnaceae, Costaceae, Lamiaceae and Tiliaceae with one genus each. A. barteri had the highest (12.89) relative abundance while the least (0.05) were Vernonia hyminolepis, Luffa aegyptiaca and Dryopteris felixmas. 
Table 1 Macrophtes of different streams in Buea

\begin{tabular}{|c|c|c|c|c|c|c|c|c|c|c|}
\hline \multirow[b]{2}{*}{ Species* } & \multicolumn{2}{|c|}{ Nange } & \multicolumn{2}{|c|}{ Mile 17} & \multicolumn{2}{|c|}{ Ndongo } & \multicolumn{2}{|l|}{ Bulu } & \multicolumn{2}{|c|}{ Wongangjio } \\
\hline & Freq & $\begin{array}{l}\text { Rel } \\
\text { Abun }\end{array}$ & Freq & $\begin{array}{l}\text { Rel } \\
\text { Abun }\end{array}$ & Freq & $\begin{array}{l}\text { Rel } \\
\text { Abun }\end{array}$ & Freq & $\begin{array}{l}\text { Rel } \\
\text { Abun }\end{array}$ & Freq & $\begin{array}{l}\text { Rel } \\
\text { Abun }\end{array}$ \\
\hline \multicolumn{11}{|l|}{ Acanthaceae } \\
\hline Asystasia gangetica (L.) T. Anderson & 1.00 & 0.01 & 12.00 & 0.66 & 19.00 & 2.27 & 34.00 & 6.65 & 39.00 & 3.32 \\
\hline Asystasia intrusa Nees & 12.00 & 4.48 & 19.00 & 10.45 & 2.00 & 0.05 & 0.00 & 0.00 & 0.00 & 0.00 \\
\hline Brillantaisia nitens Lindau & 0.00 & 0.00 & 0.00 & 0.00 & 17.00 & 2.62 & 2.00 & 0.16 & 3.00 & 0.28 \\
\hline Eremomastax speciosa (Hochst.) Cufod & 0.00 & 0.00 & 4.00 & 0.35 & 1.00 & 0.27 & 0.00 & 0.00 & 4.00 & 0.75 \\
\hline Justicia carnea Lindl. & 0.00 & 0.00 & 0.00 & 0.00 & 1.00 & 0.70 & 0.00 & 0.00 & 0.00 & 0.00 \\
\hline Ruellia strepens L. & 0.00 & 0.00 & 0.00 & 0.00 & 5.00 & 0.27 & 6.00 & 3.37 & 9.00 & 2.99 \\
\hline Ruellia prostrata Poir. & 0.00 & 0.00 & 0.00 & 0.00 & 0.00 & 0.00 & 0.00 & 0.00 & 1.00 & 2.99 \\
\hline Strobilanthes heyneanus Nees & 0.00 & 0.00 & 0.00 & 0.00 & 0.00 & 0.00 & 0.00 & 0.00 & 4.00 & 3.50 \\
\hline \multicolumn{11}{|l|}{ Amaranthaceae } \\
\hline Achyranthes aspera L. & 2.00 & 0.72 & 1.00 & 0.14 & 9.00 & 0.72 & 0.00 & 0.00 & 7.00 & 0.98 \\
\hline Alternanthera sessilis (L.) R.Br. ex DC & 0.00 & 0.00 & 0.00 & 0.00 & 20.00 & 3.82 & 6.00 & 2.16 & 0.00 & 0.00 \\
\hline Amaranthus blitum L. & 0.00 & 0.00 & 2.00 & 0.24 & 7.00 & 0.96 & 12.00 & 4.81 & 2.00 & 0.31 \\
\hline Amaranthus hybridus L. & 0.00 & 0.00 & 1.00 & 0.04 & 1.00 & 0.03 & 0.00 & 0.00 & 0.00 & 0.00 \\
\hline Amaranthus spinosa L. & 0.00 & 0.00 & 0.00 & 0.00 & 2.00 & 0.05 & 0.00 & 0.00 & 0.00 & 0.00 \\
\hline \multicolumn{11}{|l|}{ Amaryllidaceae } \\
\hline Crinum calamistratum Bogner \& Heine & 2.00 & 0.07 & 0.00 & 0.00 & 0.00 & 0.00 & 0.00 & 0.00 & 0.00 & 0.00 \\
\hline \multicolumn{11}{|l|}{ Araceae } \\
\hline Alocasia cucullata (Lour.) Schott & 0.00 & 0.00 & 0.00 & 0.00 & 1.00 & 0.03 & 0.00 & 0.00 & 0.00 & 0.00 \\
\hline Alocasia macrorrhizos (L.) G.Don & 0.00 & 0.00 & 2.00 & 0.69 & 2.00 & 0.11 & 4.00 & 0.48 & 0.00 & 0.00 \\
\hline Anubias barteri Schott. & 9.00 & 6.96 & 1.00 & 0.04 & 0.00 & 0.00 & 0.00 & 0.00 & 13.00 & 12.89 \\
\hline Colocasia esculenta (L.) Schott & 3.00 & 0.06 & 5.00 & 0.45 & 1.00 & 0.03 & 2.00 & 0.56 & 0.00 & 0.00 \\
\hline
\end{tabular}


Anyinkeng et al. / World Journal of Advanced Research and Reviews, 2020, 05(01), 001-015

\begin{tabular}{|c|c|c|c|c|c|c|c|c|c|c|}
\hline \multirow[b]{2}{*}{ Species* } & \multicolumn{2}{|c|}{ Nange } & \multicolumn{2}{|c|}{ Mile 17} & \multicolumn{2}{|c|}{ Ndongo } & \multicolumn{2}{|l|}{ Bulu } & \multicolumn{2}{|c|}{ Wongangjio } \\
\hline & Freq & $\begin{array}{l}\text { Rel } \\
\text { Abun }\end{array}$ & Freq & $\begin{array}{l}\text { Rel } \\
\text { Abun }\end{array}$ & Freq & $\begin{array}{l}\text { Rel } \\
\text { Abun }\end{array}$ & Freq & $\begin{array}{l}\text { Rel } \\
\text { Abun }\end{array}$ & Freq & $\begin{array}{l}\text { Rel } \\
\text { Abun }\end{array}$ \\
\hline Dieffenbachia picta Schott & 0.00 & 0.00 & 1.00 & 0.04 & 1.00 & 0.08 & 0.00 & 0.00 & 0.00 & 0.00 \\
\hline Xanthosoma sagittifolium (L.) Schott & 0.00 & 0.00 & 3.00 & 0.28 & 6.00 & 0.67 & 9.00 & 0.72 & 0.00 & 0.00 \\
\hline \multicolumn{11}{|l|}{ Arecaceae } \\
\hline Raphia farinifera (Gaertn.) Hyl. & 1.00 & 0.01 & 0.00 & 0.00 & 0.00 & 0.00 & 0.00 & 0.00 & 0.00 & 0.00 \\
\hline \multicolumn{11}{|l|}{ Asparagaceae } \\
\hline Dracaena sp & 0.00 & 0.00 & 0.00 & 0.00 & 1.00 & 0.03 & 0.00 & 0.00 & 0.00 & 0.00 \\
\hline \multicolumn{11}{|l|}{ Asteraceae } \\
\hline Ageratina adenophora (Spreng.) King \& H.Rob. & 8.00 & 2.47 & 13.00 & 1.90 & 0.00 & 0.00 & 0.00 & 0.00 & 0.00 & 0.00 \\
\hline Ageratum conyzoides L. & 9.00 & 2.31 & 34.00 & 10.41 & 17.00 & 5.29 & 11.00 & 5.13 & 18.00 & 9.48 \\
\hline Bidens pilosa $\mathrm{L}$. & 0.00 & 0.00 & 0.00 & 0.00 & 0.00 & 0.00 & 0.00 & 0.00 & 1.00 & 0.19 \\
\hline Chromolaena odorata (L.) King \& H.E. Robins & 11.00 & 0.48 & 0.00 & 0.00 & 0.00 & 0.00 & 0.00 & 0.00 & 2.00 & 0.09 \\
\hline Conyza boriensis (L.) Cronq. & 1.00 & 0.01 & 0.00 & 0.00 & 0.00 & 0.00 & 0.00 & 0.00 & 0.00 & 0.00 \\
\hline Crassocephalum crepidioides (Benth.) S.Moore & 0.00 & 0.00 & 2.00 & 0.48 & 4.00 & 0.37 & 1.00 & 0.16 & 4.00 & 0.19 \\
\hline Emilia coccinea (Sims) G. Don & 1.00 & 0.10 & 5.00 & 0.17 & 1.00 & 0.11 & 2.00 & 0.40 & 2.00 & 0.09 \\
\hline Galingsoga quadriradiata Ruiz \& Pav & 0.00 & 0.00 & 0.00 & 0.00 & 3.00 & 0.40 & 5.00 & 2.08 & 0.00 & 0.00 \\
\hline Synedrella nodiflora (L.) Gaertn & 0.00 & 0.00 & 2.00 & 0.24 & 15.00 & 2.97 & 8.00 & 4.09 & 2.00 & 0.09 \\
\hline Tithonia diversifolia (Hemsl.) A.Gray & 0.00 & 0.00 & 0.00 & 0.00 & 0.00 & 0.00 & 22.00 & 10.34 & 0.00 & 0.00 \\
\hline Vernonia amygdalina Delile & 1.00 & 0.01 & 1.00 & 0.04 & 1.00 & 0.03 & 0.00 & 0.00 & 0.00 & 0.00 \\
\hline Vernonia hyminolepis A. Rich. & 0.00 & 0.00 & 1.00 & 0.04 & 3.00 & 0.13 & 0.00 & 0.00 & 1.00 & 0.05 \\
\hline \multicolumn{11}{|l|}{ Athyriaceae } \\
\hline Diplazium proliferum (Lam) Thouars. & 0.00 & 0.00 & 0.00 & 0.00 & 0.00 & 0.00 & 0.00 & 0.00 & 2.00 & 0.09 \\
\hline \multicolumn{11}{|l|}{ Blechnaceae } \\
\hline Woodwardia fimbriata $\mathrm{Sm}$. & 0.00 & 0.00 & 0.00 & 0.00 & 0.00 & 0.00 & 0.00 & 0.00 & 1.00 & 0.09 \\
\hline
\end{tabular}




\begin{tabular}{|c|c|c|c|c|c|c|c|c|c|c|}
\hline \multirow[b]{2}{*}{ Species* } & \multicolumn{2}{|c|}{ Nange } & \multicolumn{2}{|c|}{ Mile 17} & \multicolumn{2}{|c|}{ Ndongo } & \multicolumn{2}{|l|}{ Bulu } & \multicolumn{2}{|c|}{ Wongangjio } \\
\hline & Freq & $\begin{array}{l}\text { Rel } \\
\text { Abun }\end{array}$ & Freq & $\begin{array}{l}\text { Rel } \\
\text { Abun }\end{array}$ & Freq & $\begin{array}{l}\text { Rel } \\
\text { Abun }\end{array}$ & Freq & $\begin{array}{l}\text { Rel } \\
\text { Abun }\end{array}$ & Freq & $\begin{array}{l}\text { Rel } \\
\text { Abun }\end{array}$ \\
\hline \multicolumn{11}{|l|}{ Brassicaceae } \\
\hline Rorippa nasturtium- aquaticum R.Br. & 13.00 & 24.05 & 0.00 & 0.00 & 0.00 & 0.00 & 0.00 & 0.00 & 5.00 & 5.37 \\
\hline \multicolumn{11}{|l|}{ Cannaceae } \\
\hline Canna. indica L. & 0.00 & 0.00 & 0.00 & 0.00 & 1.00 & 0.53 & 0.00 & 0.00 & 0.00 & 0.00 \\
\hline \multicolumn{11}{|l|}{ Commelinaceae } \\
\hline Aneilema umbrosum (Vahl) Kunth & 0.00 & 0.00 & 0.00 & 0.00 & 11.00 & 2.86 & 1.00 & 0.16 & 5.00 & 0.42 \\
\hline Commelina benghalensis L. & 43.00 & 1.06 & 42.00 & 2.15 & 45.00 & 5.13 & 41.00 & 8.57 & 39.00 & 3.36 \\
\hline \multicolumn{11}{|l|}{ Convolvulaceae } \\
\hline Ipomoea alba L. & 0.00 & 0.00 & 0.00 & 0.00 & 0.00 & 0.00 & 8.00 & 1.76 & 0.00 & 0.00 \\
\hline Ipomoea batatas (L.) Lam. & 0.00 & 0.00 & 17.00 & 0.93 & 21.00 & 2.06 & 23.00 & 4.09 & 0.00 & 0.00 \\
\hline Ipomoea muricata (L.) Jacq. & 0.00 & 0.00 & 0.00 & 0.00 & 13.00 & 1.76 & 26.00 & 7.13 & 36.00 & 6.02 \\
\hline Ipomoea purpurea (L.) Roth & 10.00 & 0.30 & 0.00 & 0.00 & 11.00 & 0.67 & 0.00 & 0.00 & 21.00 & 6.16 \\
\hline Ipomoea tiliacea (Willd.) Choisy & 0.00 & 0.00 & 54.00 & 2.80 & 3.00 & 0.32 & 0.00 & 0.00 & 0.00 & 0.00 \\
\hline \multicolumn{11}{|l|}{ Costaceae } \\
\hline Costus scaber Ruiz \& Pav. & 6.00 & 1.20 & 2.00 & 0.62 & 14.00 & 2.54 & 4.00 & 0.72 & 8.00 & 2.05 \\
\hline \multicolumn{11}{|l|}{ Crassulaceae } \\
\hline Bryophyllum pinnatum (Lam.) Oken & 1.00 & 0.09 & 0.00 & 0.00 & 0.00 & 0.00 & 0.00 & 0.00 & 0.00 & 0.00 \\
\hline \multicolumn{11}{|l|}{ Cucurbitaceae } \\
\hline Diplocyclos palmatus (L.) C.Jeffrey & 7.00 & 0.15 & 1.00 & 0.04 & 1.00 & 0.03 & 8.00 & 0.96 & 8.00 & 0.79 \\
\hline Coccinea grandis (L.) Voigt & 0.00 & 0.00 & 0.00 & 0.00 & 2.00 & 0.45 & 0.00 & 0.00 & 0.00 & 0.00 \\
\hline Luffa acutangula (L.) Roxb. & 0.00 & 0.00 & 0.00 & 0.00 & 4.00 & 0.32 & 0.00 & 0.00 & 10.00 & 0.98 \\
\hline Luffa aegyptiaca Mill. & 0.00 & 0.00 & 0.00 & 0.00 & 0.00 & 0.00 & 0.00 & 0.00 & 1.00 & 0.05 \\
\hline Momordica dioica Roxb. ex Willd & 0.00 & 0.00 & 0.00 & 0.00 & 15.00 & 1.26 & 0.00 & 0.00 & 13.00 & 1.82 \\
\hline
\end{tabular}


Anyinkeng et al. / World Journal of Advanced Research and Reviews, 2020, 05(01), 001-015

\begin{tabular}{|c|c|c|c|c|c|c|c|c|c|c|}
\hline \multirow[b]{2}{*}{ Species* } & \multicolumn{2}{|c|}{ Nange } & \multicolumn{2}{|c|}{ Mile 17} & \multicolumn{2}{|c|}{ Ndongo } & \multicolumn{2}{|l|}{ Bulu } & \multicolumn{2}{|c|}{ Wongangjio } \\
\hline & Freq & $\begin{array}{l}\text { Rel } \\
\text { Abun }\end{array}$ & Freq & $\begin{array}{l}\text { Rel } \\
\text { Abun }\end{array}$ & Freq & $\begin{array}{l}\text { Rel } \\
\text { Abun }\end{array}$ & Freq & $\begin{array}{l}\text { Rel } \\
\text { Abun }\end{array}$ & Freq & $\begin{array}{l}\text { Rel } \\
\text { Abun }\end{array}$ \\
\hline Momordica foetida Schumach. & 5.00 & 0.16 & 26 & 1.49 & 18.00 & 1.12 & 12.00 & 3.00 & 14.00 & 1.82 \\
\hline Sicyos angulatus $\mathrm{L}$. & 0.00 & 0.00 & 13.00 & 0.66 & 8.00 & 0.35 & 0.00 & 0.00 & 0.00 & 0.00 \\
\hline \multicolumn{11}{|l|}{ Cyperaceae } \\
\hline Cyperus erythrorhizos Muhl. & 5.00 & 0.46 & 7.00 & 3.84 & 1.00 & 0.05 & 0.00 & 0.00 & 2.00 & 0.37 \\
\hline Cyperus oxylepis Nees ex Steud & 1.00 & 0.04 & 0.00 & 0.00 & 0.00 & 0.00 & 0.00 & 0.00 & 0.00 & 0.00 \\
\hline Kyllinga gracillima Miq. & 1.00 & 0.07 & 0.00 & 0.00 & 2.00 & 0.21 & 0.00 & 0.00 & 1.00 & 0.47 \\
\hline \multicolumn{11}{|l|}{ Dryopteridaceae } \\
\hline Dryopteris cochleata (D. Don) C. Chr. & 0.00 & 0.00 & 0.00 & 0.00 & 0.00 & 0.00 & 0.00 & 0.00 & 8.00 & 1.17 \\
\hline Dryopteris cycadina (Franch. \& Sav.) C. Chr. & 3.00 & 0.12 & 0.00 & 0.00 & 0.00 & 0.00 & 3.00 & 0.64 & 12.00 & 1.20 \\
\hline Dryopteris felixmas (L.) Schott & 2.00 & 0.04 & 0.00 & 0.00 & 1.00 & 0.11 & 0.00 & 0.00 & 1.00 & 0.05 \\
\hline \multicolumn{11}{|l|}{ Euphorbiaceae } \\
\hline Euphorbia dentata Michx & 0.00 & 0.00 & 0.00 & 0.00 & 2.00 & 0.05 & 0.00 & 0.00 & 0.00 & 0.00 \\
\hline \multicolumn{11}{|l|}{ Fabaceae } \\
\hline Puereria phaseoloides (Roxb.) Benth. & 6.00 & 0.20 & 14.00 & 0.90 & 8.00 & 0.40 & 4.00 & 0.64 & 0.00 & 0.00 \\
\hline Vigna radiata (L.) R. Wilczek & 5.00 & 0.12 & 2.00 & 0.07 & 4.00 & 0.96 & 0.00 & 0.00 & 0.00 & 0.00 \\
\hline \multicolumn{11}{|l|}{ Heliconiaceae } \\
\hline Heliconia marginata (Greigg) Pittier & 0.00 & 0.00 & 1.00 & 0.04 & 0.00 & 0.00 & 0.00 & 0.00 & 0.00 & 0.00 \\
\hline \multicolumn{11}{|l|}{ Hydrocharitaceae } \\
\hline Vallisneria spiralis $L$. & 18.00 & 40.98 & 0.00 & 0.00 & 0.00 & 0.00 & 0.00 & 0.00 & 0.00 & 0.00 \\
\hline \multicolumn{11}{|l|}{ Lamiaceae } \\
\hline Plectranthus aboinicus (Lour.) Spreng & 0.00 & 0.00 & 0.00 & 0.00 & 0.00 & 0.00 & 0.00 & 0.00 & 3.00 & 1.54 \\
\hline Plectranthus monostachyus (P.Beauv.) B.J.Pollard & 1.00 & 0.02 & 1.00 & 0.14 & 1.00 & 0.03 & 0.00 & 0.00 & 0.00 & 0.00 \\
\hline \multicolumn{11}{|l|}{ Malvaceae } \\
\hline Abelmoschus esculentus (L).Moench & 0.00 & 0.00 & 1.00 & 0.04 & 0.00 & 0.00 & 0.00 & 0.00 & 0.00 & 0.00 \\
\hline
\end{tabular}




\begin{tabular}{|c|c|c|c|c|c|c|c|c|c|c|}
\hline \multirow[b]{2}{*}{ Species* } & \multicolumn{2}{|c|}{ Nange } & \multicolumn{2}{|c|}{ Mile 17} & \multicolumn{2}{|c|}{ Ndongo } & \multicolumn{2}{|l|}{ Bulu } & \multicolumn{2}{|c|}{ Wongangjio } \\
\hline & Freq & $\begin{array}{l}\text { Rel } \\
\text { Abun }\end{array}$ & Freq & $\begin{array}{l}\text { Rel } \\
\text { Abun }\end{array}$ & Freq & $\begin{array}{l}\text { Rel } \\
\text { Abun }\end{array}$ & Freq & $\begin{array}{l}\text { Rel } \\
\text { Abun }\end{array}$ & Freq & $\begin{array}{l}\text { Rel } \\
\text { Abun }\end{array}$ \\
\hline \multicolumn{11}{|l|}{ Melastomataceae } \\
\hline Tristemma mauritianum J.F. Gmel. & 0.00 & 0.00 & 0.00 & 0.00 & 0.00 & 0.00 & 0.00 & 0.00 & 1.00 & 0.19 \\
\hline \multicolumn{11}{|l|}{ Mimosaceae } \\
\hline Mimosa pudica L. & 0.00 & 0.00 & 4.00 & 0.14 & 0.00 & 0.00 & 0.00 & 0.00 & 0.00 & 0.00 \\
\hline \multicolumn{11}{|l|}{ Musaceae } \\
\hline Ensete ventricosum (Welw.) Cheesman & 0.00 & 0.00 & 0.00 & 0.00 & 1.00 & 0.19 & 0.00 & 0.00 & 0.00 & 0.00 \\
\hline Musa paradisiaca $L$ & 0.00 & 0.00 & 1.00 & 0.04 & 0.00 & 0.00 & 0.00 & 0.00 & 0.00 & 0.00 \\
\hline \multicolumn{11}{|l|}{ Nephrolepidaceae } \\
\hline Nephrolepis biserrata (Sw.) Schott & 8.00 & 0.74 & 0.00 & 0.00 & 0.00 & 0.00 & 0.00 & 0.00 & 2.00 & 0.09 \\
\hline Nephrolepis cordifolia (L.) K. Presl & 1.00 & 0.07 & 0.00 & 0.00 & 0.00 & 0.00 & 0.00 & 0.00 & 0.00 & 0.00 \\
\hline \multicolumn{11}{|l|}{ Onagraceae } \\
\hline Ludwigia adscendens (L.) H.Hara & 2.00 & 0.16 & 0.00 & 0.00 & 0.00 & 0.00 & 0.00 & 0.00 & 0.00 & 0.00 \\
\hline Ludwigia alternifolia $L$. & 0.00 & 0.00 & 0.00 & 0.00 & 0.00 & 0.00 & 1.00 & 0.24 & 0.00 & 0.00 \\
\hline Ludwigia longifolia (DC.) H. Hara & 1.00 & 0.07 & 0.00 & 0.00 & 0.00 & 0.00 & 0.00 & 0.00 & 0.00 & 0.00 \\
\hline Ludwigia peruviana (L.) H. Hara & 10.00 & 0.41 & 17.00 & 1.83 & 2.00 & 1.26 & 1.00 & 0.32 & 0.00 & 0.00 \\
\hline Ludwigia stolonifera (Guillemin \& al.) P. H. Raven & 11.00 & 0.59 & 3.00 & 0.623 & 2.00 & 0.48 & 1.00 & 0.24 & 0.00 & 0.00 \\
\hline \multicolumn{11}{|l|}{ Oxalidaceae } \\
\hline Oxalis simplex Salter & 0.00 & 0.00 & 0.00 & 0.00 & 2.00 & 0.08 & 1.00 & 0.08 & 0.00 & 0.00 \\
\hline \multicolumn{11}{|l|}{ Piperaceae } \\
\hline Peperomia pellucida Kunth & 0.00 & 0.00 & 1.00 & 0.07 & 0.00 & 0.00 & 0.00 & 0.00 & 0.00 & 0.00 \\
\hline Piper umbellatum L. & 0.00 & 0.00 & 0.00 & 0.00 & 2.00 & 0.83 & 1.00 & 0.41 & 26.00 & 3.74 \\
\hline \multicolumn{11}{|l|}{ Poaceae } \\
\hline Axonopus compressus (Sw.) P.Beauv & 0.00 & 0.00 & 0.00 & 0.00 & 1.00 & 0.027 & 0.00 & 0.00 & 0.00 & 0.00 \\
\hline
\end{tabular}


Anyinkeng et al. / World Journal of Advanced Research and Reviews, 2020, 05(01), 001-015

\begin{tabular}{|c|c|c|c|c|c|c|c|c|c|c|}
\hline \multirow[b]{2}{*}{ Species* } & \multicolumn{2}{|c|}{ Nange } & \multicolumn{2}{|c|}{ Mile 17} & \multicolumn{2}{|c|}{ Ndongo } & \multicolumn{2}{|l|}{ Bulu } & \multicolumn{2}{|c|}{ Wongangjio } \\
\hline & Freq & $\begin{array}{l}\text { Rel } \\
\text { Abun }\end{array}$ & Freq & $\begin{array}{l}\text { Rel } \\
\text { Abun }\end{array}$ & Freq & $\begin{array}{l}\text { Rel } \\
\text { Abun }\end{array}$ & Freq & $\begin{array}{l}\text { Rel } \\
\text { Abun }\end{array}$ & Freq & $\begin{array}{l}\text { Rel } \\
\text { Abun }\end{array}$ \\
\hline Coix lacryma-jobi $L$. & 0.00 & 0.00 & 0.00 & 0.00 & 1.00 & 0.08 & 0.00 & 0.00 & 0.00 & 0.00 \\
\hline Dactyoctenium aegyptium (L.) Beauv. & 11.00 & 0.38 & 0.00 & 0.00 & 4.00 & 0.51 & 0.00 & 0.00 & 0.00 & 0.00 \\
\hline Echinochloa cruss-galli (L.) Beauv & 0.00 & 0.00 & 0.00 & 0.00 & 4.00 & 0.67 & 0.00 & 0.00 & 0.00 & 0.00 \\
\hline Echinochloa pyramidalis (Lam.) Hitchc. \& Chase & 1.00 & 0.12 & 52.00 & 31.06 & 3.00 & 13.20 & 0.00 & 0.00 & 0.00 & 0.00 \\
\hline Eleusine indica (L.) Gaertn & 0.00 & 0.00 & 0.00 & 0.00 & 1.00 & 0.21 & 1.00 & 0.08 & 0.00 & 0.00 \\
\hline Microstegium vinineum (Trin.) A. Camus & 0.00 & 0.00 & 0.00 & 0.00 & 3.00 & 0.29 & 4.00 & 0.72 & 0.00 & 0.00 \\
\hline Panicum maximum Jacq. & 0.00 & 0.00 & 0.00 & 0.00 & 4.00 & 0.86 & 1.00 & 0.16 & 0.00 & 0.00 \\
\hline Panicum sellowii Nees & 2.00 & 0.07 & 0.00 & 0.00 & 1.00 & 0.03 & 0.00 & 0.00 & 13.00 & 1.31 \\
\hline Paspalum fimbriatum Kunth & 2.00 & 0.10 & 1.00 & 0.24 & 6.00 & 0.96 & 4.00 & 5.13 & 9.00 & 3.74 \\
\hline Pennesetum purpureum Schumach & 15.00 & 1.36 & 45.00 & 17.12 & 22.00 & 17.24 & 14.00 & 10.66 & 6.00 & 1.59 \\
\hline Sacharrum officinalis $L$. & 0.00 & 0.00 & 1.00 & 0.04 & 0.00 & 0.00 & 3.00 & 0.64 & 0.00 & 0.00 \\
\hline Zea mays $L$. & 0.00 & 0.00 & 3.00 & 0.17 & 0.00 & 0.00 & 0.00 & 0.00 & 0.00 & 0.00 \\
\hline \multicolumn{11}{|l|}{ Polygonaceae } \\
\hline Polygonum hydropiper $L$. & 0.00 & 0.00 & 0.00 & 0.00 & 4.00 & 0.96 & 0.00 & 0.00 & 0.00 & 0.00 \\
\hline Polygonum hydropiperiodes Michx. & 7.00 & 0.38 & 1.00 & 0.17 & 0.00 & 0.00 & 0.00 & 0.00 & 0.00 & 0.00 \\
\hline Polygonum persicaria L. & 22.00 & 7.46 & 1.00 & 0.14 & 0.00 & 0.00 & 0.00 & 0.00 & 0.00 & 0.00 \\
\hline \multicolumn{11}{|l|}{ Portulacaceae } \\
\hline Talinum triangulare (Jacq.) Willd & 0.00 & 0.00 & 2.00 & 0.83 & 1.00 & 0.24 & 0.00 & 0.00 & 0.00 & 0.00 \\
\hline \multicolumn{11}{|l|}{ Sellaginellaceae } \\
\hline Sellaginella kraussiana (Kunze) A. Braun & 0.00 & 0.00 & 0.00 & 0.00 & 2.00 & 0.08 & 0.00 & 0.00 & 0.00 & 0.00 \\
\hline \multicolumn{11}{|l|}{ Solanaceae } \\
\hline Brugmansia arbora (L.) Sweet & 4.00 & 0.29 & 1.00 & 0.07 & 19.00 & 2.97 & 11.00 & 2.72 & 15.00 & 3.27 \\
\hline Solanum carolinense L. & 0.00 & 0.00 & 0.00 & 0.00 & 0.00 & 0.00 & 0.00 & 0.00 & 1.00 & 0.05 \\
\hline Solanum nigrum $L$. & 0.00 & 0.00 & 5.00 & 3.22 & 2.00 & 0.11 & 0.00 & 0.00 & 0.00 & 0.00 \\
\hline
\end{tabular}


Anyinkeng et al. / World Journal of Advanced Research and Reviews, 2020, 05(01), 001-015

\begin{tabular}{|c|c|c|c|c|c|c|c|c|c|c|}
\hline \multirow[b]{2}{*}{ Species* } & \multicolumn{2}{|c|}{ Nange } & \multicolumn{2}{|c|}{ Mile 17} & \multicolumn{2}{|c|}{ Ndongo } & \multicolumn{2}{|l|}{ Bulu } & \multicolumn{2}{|c|}{ Wongangjio } \\
\hline & Freq & $\begin{array}{l}\text { Rel } \\
\text { Abun }\end{array}$ & Freq & $\begin{array}{l}\text { Rel } \\
\text { Abun }\end{array}$ & Freq & $\begin{array}{l}\text { Rel } \\
\text { Abun }\end{array}$ & Freq & $\begin{array}{l}\text { Rel } \\
\text { Abun }\end{array}$ & Freq & $\begin{array}{l}\text { Rel } \\
\text { Abun }\end{array}$ \\
\hline \multicolumn{11}{|l|}{ Tiliaceae } \\
\hline Triumfetta cordifolia A. Rich. & 1.00 & 0.04 & 4.00 & 0.73 & 3.00 & 1.23 & 0.00 & 0.00 & 26.00 & 5.79 \\
\hline \multicolumn{11}{|l|}{ Urticaceae } \\
\hline Boehmeria cylindrica (L.) Swartz & 6.00 & 0.30 & 0.00 & 0.00 & 0.00 & 0.00 & 2.00 & 0.24 & 2.00 & 6.68 \\
\hline Fleurya aestuans (L.) Gaudich & 5.00 & 0.39 & 22.00 & 3.18 & 32.00 & 11.57 & 19.00 & 8.01 & 30.00 & 0.28 \\
\hline Parietaria officinalis $L$. & 0.00 & 0.00 & 0.00 & 0.00 & 5.00 & 0.35 & 3.00 & 1.60 & 0.00 & 0.00 \\
\hline Pilea pumila (L.) A. Gray & 0.00 & 0.00 & 0.00 & 0.00 & 5.00 & 2.38 & 0.00 & 0.00 & 0.00 & 0.00 \\
\hline Urera hypselodendron (Hochst. ex A. Rich.) Wedd. & 6.00 & 0.22 & 0.00 & 0.00 & 0.00 & 0.00 & 0.00 & 0.00 & 10.00 & 1.26 \\
\hline
\end{tabular}

Note: *Frequency of occurrence (Freq) was calculated as the number of times a species was encountered in the stream. Relative abundance (Rel Abun) was calculated as the percentage of individuals of a particular species over the total number of individuals of all species. 
Overall, Poaceae and Asteraceae were the most abundant in the different streams. Plants in these families have tiny feathery/light seeds which are easily dispersed by wind and insects. The variability in the relative abundances of the different macrophytes for the different streams could be associated to the fact that some species such as $P$. purpureum are able to survive under both wetland and dry land conditions.

The highest number of families (26), genera (58) and species (70) were obtained in Ndongo while the least of these taxa were obtained in Bulu (16, 35 and 39, respectively) (Figure 2).

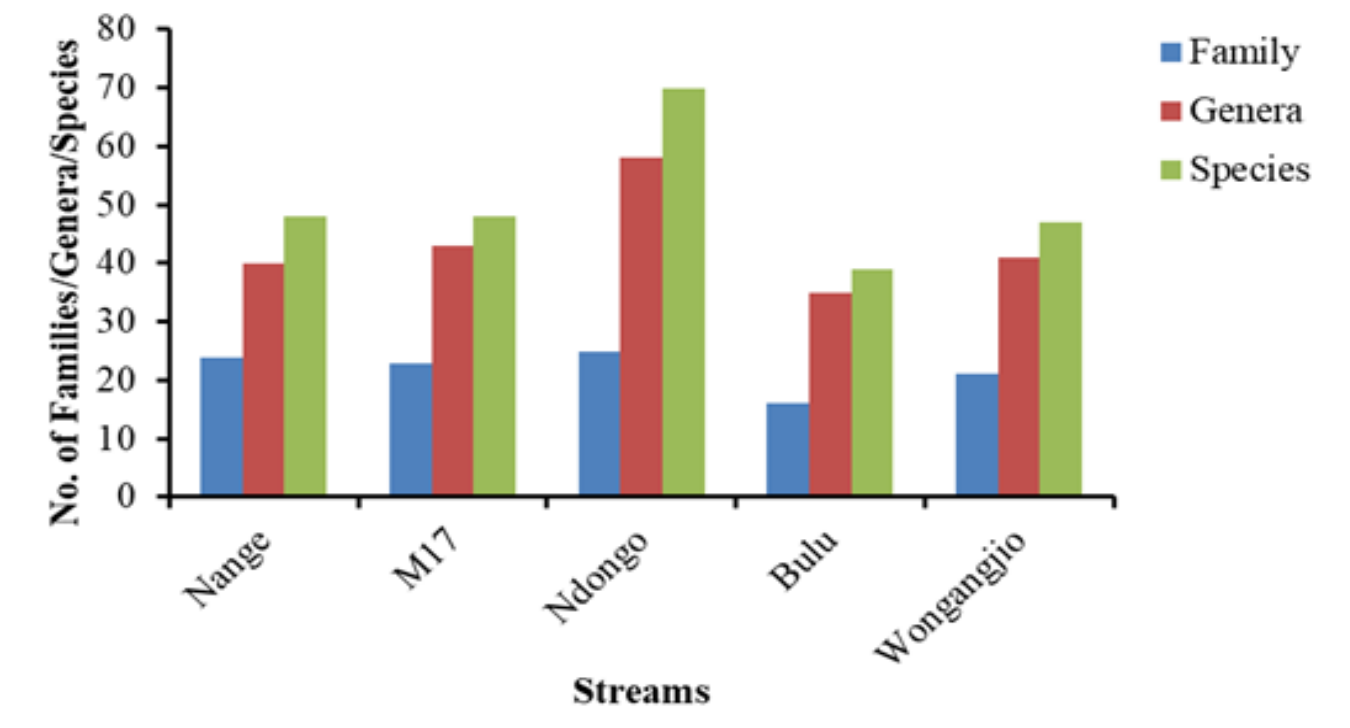

Figure 2 Macrophyte occurrence in different streams in Buea

Fourteen families, 28 genera and 44 species were specific to particular streams (Figure 3). Nange and Ndongo had the highest number (4) of unique families while Bulu had none. Across the unique genera and species, Ndongo had the highest number (10 and 13 respectively) while the least were in Bulu ( 1 and 3 respectively).The high number of unique species in Ndongo is accounted for by the presence of species which are not obligate macrophytes, brought about by anthropogenic impacts, in line with the findings of Fonkou et al. [6] in Yaounde and Tita [13] in Foumbot, other areas in Cameroon.

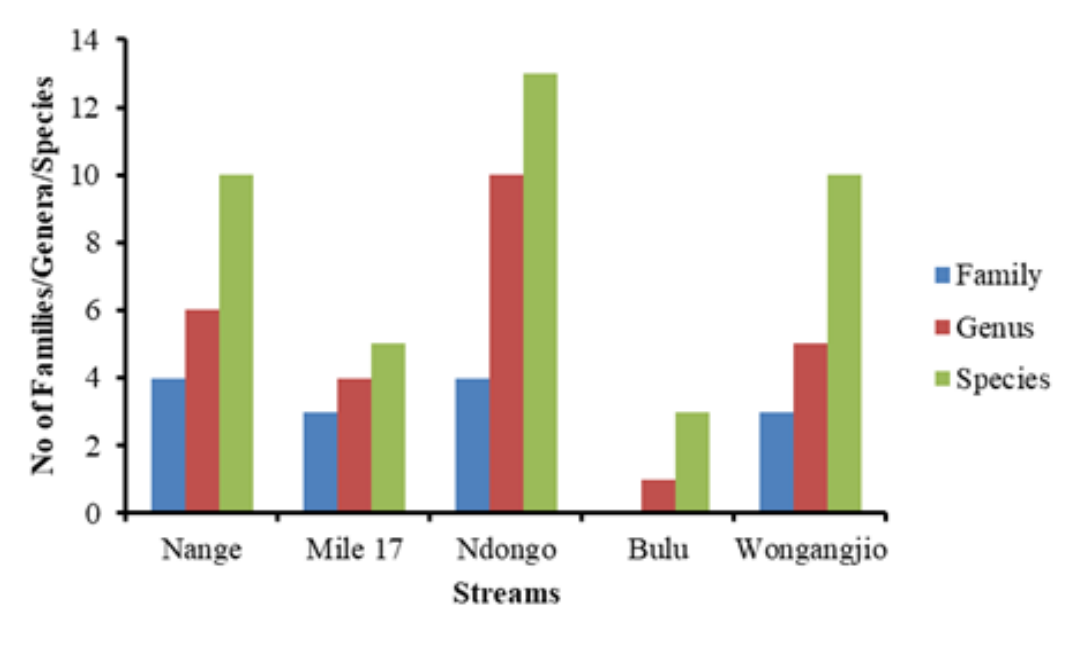

Figure 3 Unique macrophytes in different streams in Buea

The highest diversity index (0.945) was in Wongangjio while the least (0.760) was in Nange (Table 2), suggesting that waste discharge from car wash might influence species diversity negatively. The highest species richness index (1.144) was in Ndongo while the least (0.577) was in Nange. 
Anyinkeng et al. / World Journal of Advanced Research and Reviews, 2020, 05(01), 001-015

Table 2 Diversity and species richness indices of macrophytes in different streams in Buea

\begin{tabular}{lcc}
\hline Stream & Simpson's diversity index & Species richness index (D) \\
\hline Nange & 0.760 & 0.577 \\
M17 & 0.846 & 0.893 \\
Ndongo & 0.925 & 1.144 \\
Bulu & 0.939 & 1.104 \\
Wongangjio & 0.945 & 1.016 \\
\hline
\end{tabular}

The highest Sorensen similarity index (0.606) was between Ndongo and Bulu while the least (0.391) was between Nange and Bulu (Table 3) possible because the two streams Ndongo and Bulu have similar anthropogenic influence.

Table 3 Macrophyte Sorensen similarity indices of different streams in Buea

\begin{tabular}{llllll}
\hline & Nange & M17 & Ndongo & Bulu & Wongangjio \\
\hline M17 & 0.563 & 1.000 & & & \\
Ndongo & 0.458 & 0.593 & 1.000 & & \\
Bulu & 0.391 & 0.506 & 0.606 & 1.000 & \\
Wongangjio & 0.532 & 0.426 & 0.5 & 0.471 & 1.000 \\
\hline
\end{tabular}

The impact of agricultural activities on the natural environment includes the creation of new habitats, which is brought about by clearing and cultivation of plant species at the expense of others $[14,15,16]$. This is probably the reason for the high diversity indices encountered in the different streams except Nange. The presence of edible species such as Vernonia hyminolepis, Abelmoschus esculentus and Colocasia esculenta among the list further constitutes anthropogenic impact on vegetation structure along stream courses.

The spatial differences in species composition, diversity and richness of macrophytes observed are in conformity with the findings of Bini et al. [17], Thomaz et al [18] and Ruto et al. [19], and could also be accounted for by differences in physico-chemical properties [20] of both water and sediments of the streams.

\section{Conclusion}

Macrophyte diversity and abundance in Buea is high, composed of both submerged (unique to Nange) and emergent plants. The composition is made up of both obligate and non-obligate species as a consequence of anthropogenic impact along the stream belts. The abundance of the macrophytes varied with streams. Overall, Poaceae and Asteraceae were the most abundant due to anemophilic dispersal mechanisms. These results constitute baseline data for the area that can be exploited for further research and monitoring. 

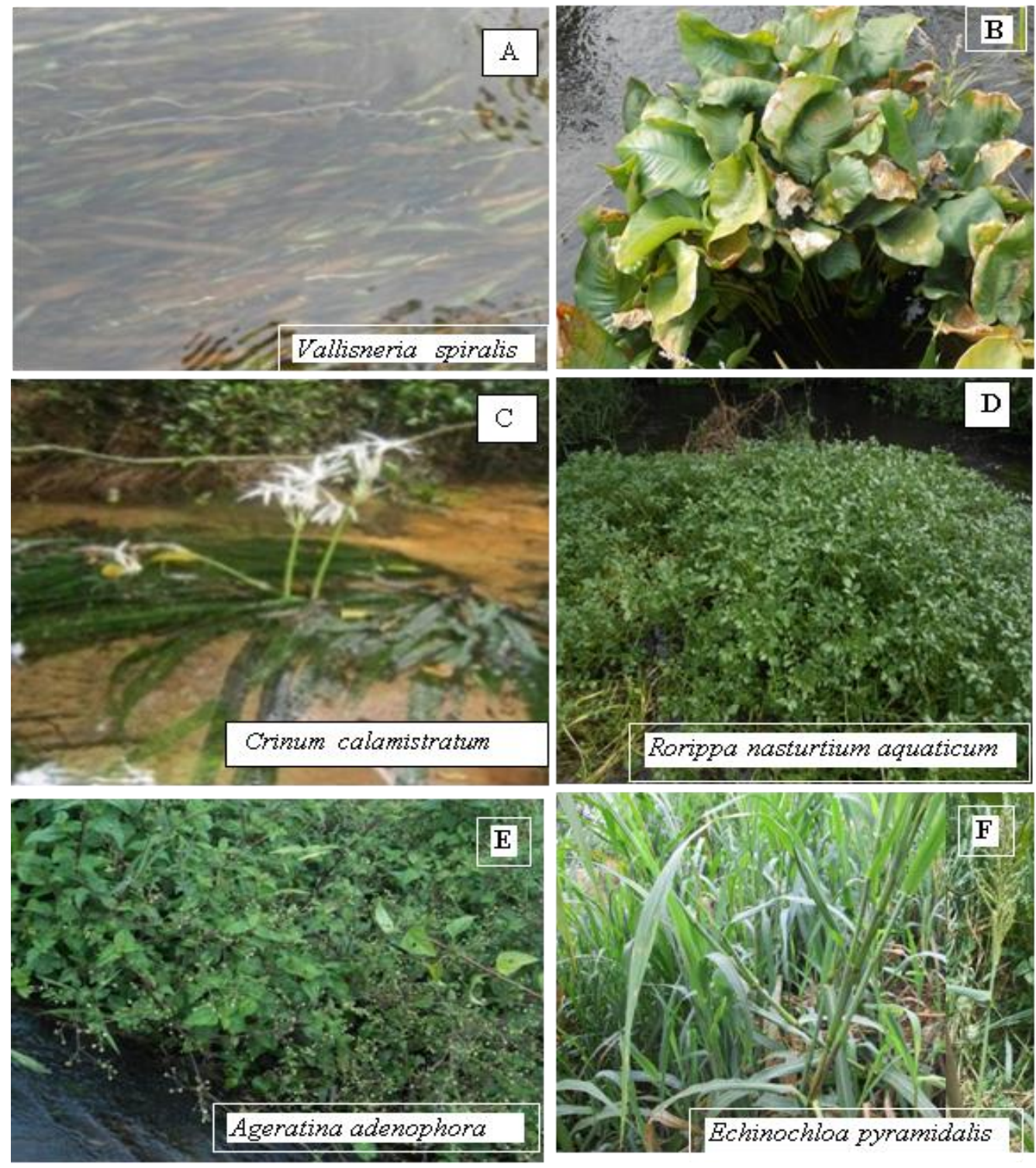

Plate 1 Submerged and Emergent macrophytes of streams in Buea Municipality, Cameroon

\section{Compliance with ethical standards}

\section{Acknowledgments}

The authors are thankful to the authority of the Limbe Botanic Garden Herbarium unit for plant identification.

\section{Disclosure of conflict of interest}

The authors do not disclose any conflict of interest.

\section{References}

[1] Tita MA, Kuitcha D, Magha A, Ndjama J and Kamgang KBV. (2012). Occurrence of macrophytes in the Mezam river system in Bamenda (Cameroon) and their role in nutrient retention. Syllabus Review, Science Series 3, 1-10.

[2] Kamel KA. (2013). Phytoremediation Potentiality of Aquatic Macrophytes in Heavy Metal Contaminated Water of EL-Temsah Lake, Ismailia, Egypt. Middle-East Journal of Scientific Research, 14, 1555-1568. 
[3] Deshmukh UB, Shende MB and Rather OS. (2016). Aquatic macrophytes biodiversity assessment from Asolamendha reservoir of Chandrapur district, Maharashtra State (India). International Journal of Applied Research, 2, 293-298.

[4] Balanson S, Walton BM, Wolin GA and Mal TK. (2005). Aquatic Macrophyte Diversity and Habitat Characterisation of the Cuyahoga River Watershed in North-eastern Ohio. The Ohio Journal of Science, 105, 88-96.

[5] Dar NA, Pandit AK and Ganai BA. (2014). Factors affecting the distribution patterns of aquatic macrophytes. Limnological Reviews, 14, 75 -81.

[6] Fonkou T, Nguetsop VF, Pinta JY, Dekoum VMA and Lekeufack M and Amougou A. (2005). Macrophyte diversity in polluted and non-polluted wetlands in Cameroon. Cameroon Journal of Experimental Biology, 1, 26-33.

[7] Fonge BA, Egbe EA, Fongod AN, Tening AS, Achu RM, Yinda GS and Mvondo- Ze A. (2012). Effects of Land Use on Macrophyte Communities and Water Quality in the Ndop Wetland Plain, Cameroon. Journal of Agriculture and Social Sciences, 12, $41-49$.

[8] Agendia P, Charbonnel Y and Valet G. (1988). Preliminary trials of several aquatic plants to treat Biyeme - Assi (Yaounde) domestic sewage. Journal of Biochemical Science, 1, 9 - 16.

[9] Kengne IM, Dodane PH, Amougou A and Kone D. (2009). Vertical - flow constructed wetlands as sustainable sanitation approach for faecal sludge dewatering in developing countries. Desalination, 248, 291 - 29.

[10] Fonkou T, Fonteh MF, Kanouo MD and Akoa A. (2010). Performances of vegetated beds with Echinochloa pyramidalis in the purification of wastewater from distillery in sub - Saharan Africa. Tropicultura, 28, 69 - 76.

[11] Folifac F, Lifongo L, Nkeng G and Gaskin S. (2009). Municipal drinking water source protection in low income countries: Case of Buea municipality-Cameroon. Journal of Ecology and Natural Environment, 1, 73-84.

[12] Pirini CB, Karagiannakidou V and Charitonidis S. (2011). Abundance, diversity and distribution of macrophytes communities in neighbouring lakes of different trophic states and morphology in North-Central Greece. Archives of Biological Science, 63, 763-774.

[13] Tita MA. (2008). Water Pollution of the Nkoup River System and its environmental impact in Foumbot, An Agricultural Town in Western Cameroon. Ph.D. thesis, University of Yaounde I, Cameroon, 145- 209.

[14] Alvarez-Yepiz JC, Martinez- Yrizar A, Burguez A and Lindquist C. (2008). Variation in vegetation structure and soil properties related to land use history of old-growth and secondary tropical dry forests in northwestern Mexico. Forest Ecology and Management, 256, 355-366.

[15] Baudry J, Burel F, Thenail C and Le Coeur D. (2000). A holistic landscape ecological study of the interactions between farming activities and ecological patterns in Brittany, France. Landscape and Urban Planning, 50, $19-28$.

[16] Aguiar FC and Ferreira MT. (2006). Riparian and aquatic vegetation in Mediterranean type streams (western Iberia). Limnetica, 25, 411 - 424.

[17] Bini LM, Thomaz SM and Souza DC. (2001). Species richness and beta-diversity of aquatic macrophytes in the Upper Parana River floodpain. Archiv fur Hydrobiology, 151, 511-525.

[18] Thomaz SM, Carvalho P, Padial AA and Kobayashi JT. (2009). Temporal and spatial patterns of aquatic macrophyte diversity in the Upper Paraná River floodplain. Brazilian Journal of Biology, 69, 617-625.

[19] Ruto WKS, Kinyamario JI, Ng'etich NK, Akunda E and Mworia JK. (2012). Plant Species Diversity and Composition of Two Wetlands in the Nairobi National Park, Kenya. Journal of Wetlands Ecology, 6, 7-15.

[20] Thomaz SM, Pagioro TA, Bini LM, Roberto MC and Rocha RRA. (2004). Limnological characterization of the aquatic environments and the influence of hydrometric levels. In: Thomaz SM, Agostinho AA and Hahn NS (Eds). The upper Parana River and its floodplain: physical aspects, ecology and conservation. Leiden, The Netherlands, Backhuy publishers, 75-102.

\section{How to cite this article}

Anyinkeng N, Mih MA and Tening AS. (2020). Diversity and abundance of macrophytes of streams under different anthropogenic influences in the Buea municipality, southwestern Cameroon. World Journal of Advanced Research and Reviews, 5(1), 01-15. 